\title{
Canales endémicos y calidad de la información para su elaboración en municipios seleccionados
}

\section{Endemic ranges and the quality of information in selected municipalities} Gisele Coutin Marie'; Elba Nieves Pérez Moreno"'; Leana Margarita Labrada
Moreno'"'; Oney Terry Villa'I'; Neylim Blanco Hernández"

\author{
' Máster en Informática. Especialista de II Grado de Bioestadística. Unidad Nacional \\ de Análisis y Tendencias en Salud. Ministerio de Salud Pública. La Habana, Cuba. \\ "Especialista de II Grado de Higiene y Epidemiología. Unidad de Análisis y \\ Tendencias en Salud de la provincia de Pinar del Río. Pinar del Río, Cuba. \\ III Residente de Bioestadística de Segundo Año. Unidad Nacional de Análisis y \\ Tendencias en Salud / Ministerio de Salud Pública. La Habana, Cuba. \\ IV Máster en Infectología y Enfermedades Tropicales. Unidad Nacional de Análisis y \\ Tendencias en Salud / Ministerio de Salud Pública. La Habana, Cuba.
}

\section{RESUMEN}

La detección precoz del comportamiento anormal de enfermedades transmisibles depende de la calidad y oportunidad de la información disponible y de métodos eficientes. En Cuba generalmente se basa en análisis de series cronológicas y canales endémicos para vigilancia semanal o mensual desde el nivel primario de atención. En el país no se ha evaluado suficientemente la calidad de las series cronológicas ni los métodos empleados. En el año 2006 se realizó un estudio descriptivo en 18 unidades de análisis y tendencias municipales y en 12 áreas de salud de municipios seleccionados de Ciudad de La Habana y Pinar del Río, para evaluar la calidad de los datos utilizados en la confección de canales endémicos, identificar los principales métodos utilizados para su elaboración y personal que los analiza. Se encontró que se vigilan un número suficiente de Enfermedades de Declaración Obligatoria aunque no de manera uniforme, el soporte de conservación más utilizado en áreas de salud fue el papel, se conservan un número elevado de años en casi todas las unidades, el método más utilizado para el canal endémico es el Máximo maximorum y Mínimo minimorum y el personal que realiza el análisis está suficientemente capacitado. Las series existentes en esas unidades permiten realizar análisis apropiados y es conveniente extender la conservación digital como norma así como incrementar la preparación de profesionales dedicados a esta actividad.

Palabras clave: Canales endémicos, detección de epidemias, series de tiempo, técnicas estadísticas para la vigilancia en atención primaria de salud. 


\section{ABSTRACT}

Early detection of the anomalous behavior of communicable diseases depends on the quality and promptness of available data and of efficient methods. In Cuba, this detection generally supports on the analysis of time series and endemic ranges for weekly or monthly surveillance at primary health care. There have not been thorough assessment of the quality of times series and methods. A descriptive study was conducted in 18 municipal health analysis and trends units and in 12 health areas located in selected municipalities of the City of Havana and Pinar del Río provinces in 2006 , with the purpose of evaluating the quality of data in the preparation of endemic ranges; to identify the main methods for their preparation and the staff in charge of the analysis. It was found that an adequate number of compulsory notification diseases are under surveillance, but not in a consistent way; the most used preservation means is paperback in health areas; a high number of years in analysis are kept in almost all the units, the most common method for preparation of endemic ranges is Maximum Maximorum and Minimum Minimorum whereas the staff in charge of the analysis is properly trained. The existing time series allow making suitable analysis, so, it is desirable to increase the use of computerization to keep them as well as to raise the level of training for the professionals devoted to this task.

Key words: Endemic ranges, epidemic detection, time series, statistical techniques for surveillance at primary health care.

\section{NTRODUCCI ÓN}

La Vigilancia en Salud constituye una actividad de seguimiento, recolección sistemática, análisis e interpretación de datos sobre eventos de salud o condiciones relacionadas, cuya finalidad principal es alertar a las autoridades competentes con oportunidad suficiente para poder tomar las medidas necesarias, ya sean preventivas o de control. Esta vigilancia incluye el proceso de detección de enfermedades mediante un sistema de recolección de información estandarizado que garantice la calidad del dato, la interpretación y los análisis adecuados para las autoridades sanitarias que deben enfrentar los problemas de salud. El sistema de vigilancia debe estar estructurado de manera tal que la magnitud o el tipo de problema no impidan la respuesta adecuada y para ello debe basarse en sólidas capacidades de diagnóstico y habilidad para la detección precoz desde el primer nivel de atención. ${ }^{1,2}$

La detección precoz depende en mucho de la calidad y oportunidad de la información disponible mientras que la determinación de si una enfermedad se encuentra en epidemia o no, requiere de métodos lo suficientemente sensibles y eficientes. La mayoría de los métodos empleados en Cuba para evaluar desviaciones del comportamiento actual de un problema de salud versus su comportamiento esperado o habitual, se basan en el análisis de las series cronológicas que se conservan en las diferentes instancias del sistema de salud con las cuales se elaboran los canales endémicos empleados para la vigilancia semanal o mensual de la mayoría de las enfermedades transmisibles. ${ }^{3}$ 
El canal endémico creado por Selwyn Collins en 1932 para la vigilancia de las epidemias de influenza y muy utilizado desde entonces debido a la simplicidad de su confección e interpretación, es una herramienta que permite conocer el comportamiento y evaluar la naturaleza endémica o epidémica de una enfermedad. Constituye una representación gráfica de la incidencia actual sobre la incidencia histórica y permite detectar precozmente cifras anormalmente altas o bajas de casos de la enfermedad en estudio. ${ }^{4,5}$

Existen varios métodos para confeccionar canales endémicos y todos consisten en la obtención de una medida central que sirve como curva de expectativa y otras dos curvas que enmarcan el recorrido de fluctuación normal de la incidencia para cada uno de los intervalos de tiempo considerados, fundamentalmente semanas o meses, a partir de una serie notificada de casos en un período de 5 a 7 años. El aumento de la cantidad de años no mejora sustancialmente el análisis ya que es muy probable que se mantengan estables los factores que han generado la serie sobre todo los criterios diagnósticos, los mecanismos de notificación y registro y la endemia característica del evento. ${ }^{6-8}$

Los métodos más utilizados para elaborar canales endémicos en el país tanto por las unidades de análisis y tendencias en salud (UATS) provinciales y municipales como por las áreas de salud, emplean como medida central la mediana, dadas las características de este estadígrafo que no se ve afectado por los valores extremos de la serie y aunque se prefiere para obtener los límites de variación la utilización de los valores inframáximos y supramínimos, pues en este procedimiento se desechan las observaciones extremas superiores e inferiores lo que contribuye a incrementar la sensibilidad del canal para la detección precoz de epidemias, no todas las unidades lo realizan así. ${ }^{9,10}$ Además, en la Unidad Nacional de Análisis y Tendencias en Salud, así como en varias unidades provinciales, se ha aplicado la modelación ARI MA para obtener los valores esperados y sus límites de variación en series semanales y mensuales de diversas enfermedades transmisibles con mucho éxito, pero esta modelación es más compleja y requiere de un entrenamiento especial, por lo cual no ha podido ser extendida hasta las unidades municipales. ${ }^{11-13}$

La recolección de información de buena calidad para la confección de los canales endémicos constituye un elemento esencial para mantener la efectividad de la vigilancia. En Cuba, existen las condiciones necesarias para garantizar la calidad de esta información desde que en la década de los años 60 del pasado siglo, se dictaron las regulaciones oficiales para normar la declaración obligatoria de un conjunto de enfermedades que permitieron disponer desde entonces de un registro, cuyas garantías de integridad y oportunidad han podido ser comprobadas durante las 4 décadas transcurridas. ${ }^{14,15}$

El concepto de calidad de los datos tiene múltiples dimensiones, pero las más importantes están relacionadas con la relevancia que debe tener el dato de acuerdo a las necesidades de los usuarios y las características de la recolección de la información, entre las que se pueden señalar su validez, oportunidad, accesibilidad, interpretabilidad y coherencia. ${ }^{16,17}$ Para la realización de una vigilancia adecuada la validez y oportunidad de obtención y procesamiento de los datos resulta de cardinal importancia.

Las causas que pueden afectar la calidad de los datos para la vigilancia son múltiples, desde errores relacionados con la recolección y procesamiento manuales o automáticos de los datos, la conservación inadecuada de las series, las variaciones en los métodos de recolección y conservación hasta los ocasionados por sub registros de los eventos sujetos a notificación debidos a no registro de casos 
por parte de los profesionales encargados desde el nivel primario, variaciones en los mecanismos de notificación y otros. ${ }^{18,19}$

Entre las funciones principales de las UATS está el perfeccionamiento constante de los métodos de detección precoz así como la evaluación de los habitualmente empleados con la finalidad de ofrecer alertas cada vez más eficientes y oportunas a las autoridades del Sistema Nacional de Salud y especialmente a la atención primaria de salud.

Con el propósito de evaluar la calidad de los datos utilizados para la confección de canales endémicos, la identificación de los principales métodos utilizados para su elaboración y del personal que los analiza en municipios seleccionados del país, se realizó un estudio descriptivo durante el año 2006. En este artículo se presentan los resultados obtenidos.

\section{MÉTODOS}

Se realizó un estudio descriptivo en 18 municipios del país pertenecientes a las provincias de Pinar del Río y Ciudad de la Habana. En Pinar del Río se analizaron todos los municipios: Sandino, Mantua, Minas, Viñales, La Palma, Bahía Honda, Candelaria, San Cristóbal, Los Palacios, Consolación del Sur, Pinar del Río, San Luís, San Juan y Martínez y Guane; en la Ciudad de La Habana se escogieron de forma no aleatoria los municipios 10 de Octubre, Marianao, Centro Habana y Boyeros. En todos se visitaron las unidades municipales de análisis y tendencias en salud. También se seleccionaron no aleatoriamente las 14 áreas de salud que se muestran en el recuadro. 
Recuadro. Áreas de salud seleccionadas

\begin{tabular}{|l|c|}
\hline Áreas de salud & Municipio \\
\hline Policlínico Pasteur & 10 de Octubre \\
\hline Policlínico Lawton & 10 de Octubre \\
\hline Policlínico Salvador Allende & Boyeros \\
\hline Policlínico Mártires de Calabazar & Boyeros \\
\hline Policlínico Carlos J. Finlay & Marianao \\
\hline Policlínico 27 de Noviembre & Centro Habana \\
\hline Policlínico Nguyen Van Troi & Centro Habana \\
\hline Policlínico Reyna & San Cristóbal \\
\hline Policlínico Camilo Cienfuegos & San Cristóbal \\
\hline Policlínico Santa Cruz & Pinar del Río \\
\hline Policlínico Pedro Borras & Pinar del Río \\
\hline Policlínico Turcios Lima & Pinar del Río \\
\hline Policlínico Raúl Sánchez & \\
\hline Policlínico Hermanos Cruz & \\
\hline
\end{tabular}

En estas unidades se analizaron las series cronológicas de 16 Enfermedades de Declaración Obligatoria (EDO) seleccionadas por su mayor incidencia tradicional: enfermedades diarreicas agudas (EDA), infecciones respiratorias agudas (IRA), hepatitis virales, tuberculosis, leptospirosis, sífilis, blenorragia, condiloma acuminado, varicela, herpes zoster, escarlatina, meningitis bacterianas (MEB), meningitis virales (MEV), síndrome febril, intento suicida e intoxicaciones alimentarias. En cada serie se verificó el soporte de conservación (digital o papel), su estado de conservación, periodicidad y el número de años conservados. También se comprobó la existencia de canales endémicos, el método utilizado para su confección así como el personal que los analiza.

\section{RESULTADOS}

En la tabla 1 se observa que el $71,43 \%$ de las áreas de salud estudiadas conservaba las series cronológicas en papel, solamente 1 de ellas lo guardaba en soporte digital. En las UATS municipales se apreció que el 55,56 \% dispone de ambos medios de conservación de los documentos. En todas las unidades revisadas cuyo soporte de conservación fue papel, se trataba de documentos manuscritos llenados a lápiz que en algunos casos no estaban en buen estado. 
Tabla 1. Conservación de las series cronológicas según tipo de soporte y unidad

\begin{tabular}{|l|c|c|c|c|}
\hline \multirow{2}{*}{$\begin{array}{l}\text { Soporte de } \\
\text { Conservacion }\end{array}$} & \multicolumn{4}{|c|}{ Tipo de unidad } \\
\cline { 2 - 5 } & A.reas de salud & No. & UATS \\
\cline { 2 - 5 } & No. & 71,43 & 1 & 5,56 \\
\hline Papel & 10 & 7,14 & 7 & 38,89 \\
\hline Digital & 1 & 21,43 & 10 & 14,71 \\
\hline Ambos & 3 & 100,00 & 18 & 100,00 \\
\hline Total & 14 & & & \\
\hline
\end{tabular}

En la tabla 2 se aprecia que en la mitad de las áreas de salud se utilizaban series semanales y mensuales, y en 4 áreas $(28,57 \%)$ solamente se conservaban series mensuales. En el $88,89 \%$ de las UATS se mantenía ambos tipos de periodicidad, solamente en 2 de ellas se analizaban datos mensuales nada más.

Tabla 2. Periodicidad de las series cronológicas conservadas según unidad

\begin{tabular}{|c|c|c|c|c|}
\hline \multirow[t]{3}{*}{ Periodicidad de las series } & \multicolumn{4}{|c|}{ Tipo de unidad } \\
\hline & \multicolumn{2}{|c|}{ Áreas de salud } & \multicolumn{2}{|c|}{ UATS } \\
\hline & No. & $\%$ & No. & $\%$ \\
\hline Semanal & 3 & 21,43 & 0 & 0,00 \\
\hline Mensual & 4 & 28,57 & 2 & 11,11 \\
\hline Ambas & 7 & 50,00 & 16 & 88,89 \\
\hline Total & 14 & 100,00 & 18 & 100,00 \\
\hline
\end{tabular}

En las áreas de salud se disponía de series cronológicas para un promedio de 11 años, aunque esta cifra varió pues algunas unidades solamente tenían datos de los últimos 4 años, como es el caso de los policlínicos "27 de Noviembre" y "Nguyen Van Troi". En las UATS el promedio de conservación era de 10,4 pero se apreció gran variabilidad sobre todo en la provincia de Pinar del Río pues hay unidades en los municipios Guane y Sandino donde solo se conservaba información de los últimos 5 años (tabla 3 ).

Tabla 3. Años de información conservados según unidad

\begin{tabular}{|l|c|c|}
\hline \multicolumn{1}{|c|}{} & \multicolumn{2}{|c|}{ Tipo de unidad } \\
\cline { 2 - 3 } & Áreas de salud & UATS \\
\hline $\begin{array}{l}\text { Promedio de años } \\
\text { conservados }\end{array}$ & 11 & 10,4 \\
\hline Número mínimo & 4 & 5 \\
\hline Número máximo & 10 & 26 \\
\hline
\end{tabular}

El total de eventos controlados en esta investigación fue de 16 EDO, en la tabla 4 se aprecia que el número promedio de enfermedades que disponen de series 
cronológicas completas es similar tanto en las áreas de salud como en UATS municipales (13), aunque en las primeras existió una cifra mínima de 5 que se produjo en el policlínico "Nguyen Van Troi". Las EDO que con más frecuencia no tenían series cronológicas completas en ambos tipos de unidades eran: síndromes febriles, intento suicida e intoxicaciones alimentarias y las que existen en todas las unidades son EDA, IRA, hepatitis virales y tuberculosis.

Tabla 4. Series cronológicas de Enfermedades de Declaración Obligatoria conservadas según unidad

\begin{tabular}{|l|c|c|}
\hline \multicolumn{1}{|c|}{} & \multicolumn{2}{|c|}{ Tipo de unidad } \\
\cline { 2 - 3 } & Áreas de salud & UATS \\
\hline Promedio de series conservadas & 13 & 13 \\
\hline Número mínimo & 5 & 11 \\
\hline Número máximo & 16 & 15 \\
\hline
\end{tabular}

La elaboración de canales endémicos no se comportó de manera uniforme, en la tabla 5 se aprecia que el $42,86 \%$ de las áreas de salud no lo hacían, mientras que sí se confeccionaban en el $100 \%$ de las UATS municipales.

Tabla 5. Elaboración de canales endémicos según unidad

\begin{tabular}{|l|c|c|c|c|}
\hline \multicolumn{1}{|c|}{ Canales endémicos } & \multicolumn{3}{|c|}{ Tipo de unidad } \\
\cline { 2 - 5 } & \multicolumn{2}{|c|}{ Áreas de salud } & \multicolumn{2}{c|}{ UATS } \\
\cline { 2 - 5 } & No. & $\%$ & No. & $\%$ \\
\hline Realizados & 8 & 57,14 & 18 & 100,00 \\
\hline No realizados & 6 & 42,86 & 0 & 0,00 \\
\hline Total & 14 & 100,00 & 18 & 100,00 \\
\hline
\end{tabular}

En relación con los métodos utilizados para la elaboración de los canales endémicos, el más utilizado en ambos tipos de unidades, es el método del Máximo maximorum-Mínimo minimorum $(66,67 \%$ de las UATS y $78,57 \%$ de las áreas de salud). En la UATS municipal de San Cristóbal se construyen además corredores endémicos (tabla 6). 
Tabla 6. Métodos empleados para la elaboración de canales endémicos según unidad

\begin{tabular}{|l|c|c|c|c|}
\hline \multicolumn{1}{|l|}{ Método de elaboración } & \multicolumn{3}{|c|}{ Tipo de unidad } \\
\cline { 2 - 3 } & Áreas de salud & \multicolumn{2}{c|}{ UATS } \\
\hline & No. & $\%$ & No. & $\%$ \\
\hline \begin{tabular}{l|c|c|c|} 
Máximo maximorum - Mínimo \\
minimorum
\end{tabular} & 11 & 78,57 & 12 & 66,67 \\
\hline Cifras inframáximas y supramínimas & 3 & 21,43 & 5 & 27,78 \\
\hline Otros & 0 & 0,00 & 1 & 5,56 \\
\hline Total & 14 & 100,00 & 18 & 100,00 \\
\hline
\end{tabular}

En la mayoría de las UATS municipales investigadas el personal que confecciona y analiza la información que ofrecen los canales endémicos tiene preparación suficiente para ello, en los 4 municipios de la Ciudad de la Habana todos son médicos especialistas en higiene y epidemiología, higiene del trabajo o especialistas de medicina general integral con diplomados en epidemiología, mientras que en la provincia Pinar del Río han recibido preparación en la UATS provincial mediante un diplomado en vigilancia en salud: 8 licenciadas en enfermería, 1 médico veterinario y 3 especialistas de medicina general integral. En esta provincia, solamente en las unidades de San Cristóbal y San Luis, la actividad es realizada por un especialista de higiene y epidemiología y un máster de epidemiología, respectivamente.

En las áreas de salud la situación es también variable pues en los policlínicos de Ciudad de La Habana esta actividad se realiza por los vice directores de higiene y epidemiología que son en su mayoría médicos especialistas en epidemiología, o especialistas en medicina general integral con diplomados en epidemiología mientras que en Pinar del Río, de las 6 áreas estudiadas, solamente se realiza en los 2 policlínicos del municipio San Cristóbal por licenciadas en enfermería que han recibido un diplomado en vigilancia en salud.

\section{DISCUSIÓN}

La existencia de series cronológicas conservadas en todas las áreas constituye una garantía para la realización de la vigilancia de los eventos seleccionados, no obstante, el hecho de que en algunos de los policlínicos estas existan con una periodicidad mensual únicamente, atenta contra la oportunidad del alerta pues la detección precoz necesita de la vigilancia semanal. La conservación de las series en documentos manuscritos solamente, constituye una debilidad importante en varias áreas dado que son más propensos al deterioro, lo que le resta calidad como fuente de información para el análisis.

El número de años de los que se dispone de información ya sea semanal o mensual en casi todas las unidades es suficiente para la construcción de canales endémicos. De igual modo resultan suficientes los eventos vigilados pues en la mayoría de las áreas y en todas las UATS municipales existen las series cronológicas de todos los eventos analizados, aunque la no conservación de las series cronológicas de síndromes febriles en algunas unidades puede convertirse en un problema serio, dada la importancia que tiene este evento para alertar acerca de la aparición de enfermedades emergentes o reemergentes como plantean Oliva $M$, Troyes $L$ y otros. ${ }^{20,21}$ 
El conocimiento de la calidad de los datos que conforman las series cronológicas y la necesidad de su evaluación periódica ha motivado investigaciones en otros países aun fuera del ámbito de la salud pública. Maravall y Luna en 1999 desarrollaron en España un método automático para la detección de errores en series temporales económicas basados en modelos de pronóstico, que posteriormente ha sido utilizado también por Quirós J . en Costa Rica en el 2002. ${ }^{22,23}$ Aguilera y otros implementaron un protocolo para evaluar la calidad de los datos de las series de gripe recogidos por médicos centinelas en Europa durante el año 2002. ${ }^{24}$ Estos autores consideran de capital importancia la evaluación de la calidad de los datos y la utilización de series cronológicas para vigilancia semanal.

No obstante, en Cuba no existen muchos estudios anteriores que permitan conocer cuán buenas son las series temporales existentes para la vigilancia en el país. Díaz $\mathrm{J}$, en su evaluación de la calidad del Programa de Control de la Tuberculosis en el municipio La Lisa, La Habana en el 2001, pudo apreciar la buena calidad de las series cronológicas de este evento en las áreas de salud del citado municipio. Por su parte Guanche y otros pudieron utilizar series lo suficientemente buenas para su estudio de morbilidad y letalidad por asma bronquial en atención secundaria. ${ }^{25,26}$ Los resultados obtenidos en el presente trabajo coinciden con los de Almenares K, que utilizó en el 2005 las series cronológicas de Enfermedades Transmisibles conservadas en buen estado en el municipio Plaza de La Revolución de La Habana, para la implementación de técnicas de control de calidad. ${ }^{27}$

En cuanto a los métodos con los cuales se confeccionan los canales endémicos en las unidades estudiadas, Ilama la atención que una gran mayoría de las unidades prefieran utilizar el método del Máximo maximorum y Mínimo minimorum muy desaconsejado debido a la pérdida de sensibilidad que presenta para el alerta precoz al incluir las cifras epidémicas, aun cuando disponen de un conjunto de años suficientes para emplear otros métodos más sensibles. Aunque Torrescano y otros recomiendan la utilización de los cuartiles primero y tercero para la construcción de los límites de variación y la no inclusión de años epidémicos, la utilización de cifras inframáximas para el límite de variación superior, como utilizan la minoría de las áreas de salud y todas las UATS municipales analizadas, logra efecto similar al eliminar los valores más altos. ${ }^{28}$

Otro aspecto importante es la capacidad de análisis y comprensión de los canales endémicos puesto que un análisis epidemiológico riguroso permite perfeccionar el procedimiento de vigilancia y ofrecer la alerta oportuna, así como la identificación de problemas de investigación a nivel de las áreas. Jiménez $\mathrm{S}$. alerta acerca de las consecuencias que tiene para un sistema de vigilancia la falta de preparación adecuada para la interpretación de la información. ${ }^{29}$ Dado que en la mayoría de las unidades analizadas existen profesionales a cargo del análisis que han recibido al menos un diplomado en epidemiología o en vigilancia en salud, existe una buena capacidad para realizarlo adecuadamente.

Se puede concluir que la práctica eficiente de la vigilancia necesita de herramientas apropiadas para la detección precoz de problemas y la emisión oportuna de alertas desde el nivel primario. Esto no resulta posible sin la utilización de series cronológicas de buena calidad y métodos sensibles como la generalidad de los apreciados en este trabajo. La correcta elaboración e interpretación de los canales endémicos en la atención primaria de salud fortalece el proceso de la vigilancia a partir de este nivel.

Sin embargo, la necesidad del alerta precoz requiere de la integración de datos de diversas fuentes y deben aprovecharse las posibilidades que brinda la 
automatización ya que la utilización de métodos manuales para la confección de series y la conservación en documentos de papel manuscritos atentan contra la buena calidad de la vigilancia.

Aunque los profesionales dedicados a esta área tienen algún grado de preparación para ejecutar las tareas pues la mayoría son universitarios, profesionales propios del sector de la salud que han recibido diferentes entrenamientos, resulta necesario continuar perfeccionando su preparación para el análisis, dados los riesgos siempre crecientes que implica para la población del país, el contexto de salud mundial actual caracterizado por la reemergencia de enfermedades y el surgimiento de otras nuevas.

\section{REFERENCI AS BI BLI OGRÁFICAS}

1. Coutin Marie G. Vigilancia en salud: Apuntes sobre su desarrollo histórico [sitio en Internet]. [citado 25 Ene 2007]. Disponible en: http://www. sld.cu/galerias/pdf/sitios/vigilancia/rtv0305.pdf

2. USAID Global Health Infectious Diseases. USAID's Infectious Disease Surveillance Strategy [sitio en Internet]. [citado 25 Ene 2007]. Disponible en: http://www.usaid.gov/pop_health/id/surveillance/index.html

3. Coutin G, Borges J, Batista R, Feal P. Métodos para la Vigilancia de Eventos en Salud. Rev Cubana Hig Epidemiol. 2000; 38(3): 157-66.

4. Lesson One: Influenza Pandemics. Historical Perspectives [sitio en Internet]. [citado 12 J un 2006]. Disponible en :

http://catalyst. washington.edu/workshops/resources/text_edited.doc

5. 121 CDC. Cities Mortality Reporting System: History [sitio en Internet]. [citado 1 Oct 2007]. Disponible en URL: http://www.cdc.gov/epo/dphsi/121hist.htm

6. Ferrario C, Rial MJ, Magariños M. Confección de corredores endémicos de virus respiratorios[sitio en Internet]. [citado 2 Feb 2007]. Disponible en:

http://www.elizalde.gov.ar/novedades/end\%E9micos.pdf

7. Bortman M. Elaboración de Corredores o Canales Endémicos mediante planillas de cálculo. Rev Panam Salud Pública. 1999; 5(1):15-22.

8. Observatorio de Salud. Métodos para la confección de Canales Endémicos [sitio en Internet]. [citado 2 Feb 2007]. Disponible en :

http://www. guanajuato.gob. mx/ssg/observatorio/canal.htm

9. Coutin G. Análisis de Series temporales. Folleto para la Maestría de Salud Pública. Escuela Nacional de Salud Pública de Cuba [sitio en Internet].2021 [citado 2 Feb 2007]. Disponible en :

http://www.vcl.sld.cu/iscm/facmed/web\%20salud/materiales/seriet.htm

10. Gómez C. Métodos para la Vigilancia de eventos: Corredores endémicos. Reporte Técnico de Vigilancia [sitio en Internet]. 2000 [citado 2000]; 5(4). Disponible en: http://www.sld.cu/galerias/pdf/sitios/vigilancia/rtv0400.pdf

11. Coutin G, Sacerio Z, Hernández P, Abreu Y. Pronósticos de Infecciones Respiratorias Agudas. Cuba, 1996-2006. Reporte Técnico de Vigilancia [sitio en 
Internet]. 2006 [citado 2 Oct 2007];11(5). Disponible en :

http://www.sld.cu/galerias/pdf/sitios/vigilancia/coutin5a2006.pdf

12. Coutin G, Columbié M, Hernández P. Análisis de Hepatitis Viral en Cuba, 19772005: Pronósticos para la vigilancia semanal. Reporte Técnico de Vigilancia [sitio en Internet]. 2006 [citado 2 Oct 2007]; 11(5). Disponible en:

http://www.sld.cu/galerias/pdf/sitios/vigilancia/coutin5b2006.pdf

13. Coutin G, Hernández $P$, Columbié $M$. Tuberculosis pulmonar. Análisis de la serie y obtención de pronósticos. Cuba, 1996 _ 2004. Reporte Técnico de Vigilancia [sitio en Internet]. 2006 [citado 2 Oct 2007];11(5). Disponible en:

http://www.sld.cu/galerias/pdf/sitios/vigilancia/coutin5b2006.pdf

14. Zacca Peña E, Martínez Morales MA. Sistema de Información Estadístico de Enfermedades de Declaración Obligatoria (EDO). Revista Temas Estadísticos de Salud [sitio en Internet]. [citado 25 Ene 2007]; (1). Disponible en:

http://www.sld.cu/galerias/pdf/sitios/dne/edo.pdf

15. Ríos Massabot NE, Fernández Viera RM, Jorge Pérez ER. Los registros médicos en Cuba. Rev Cubana Salud Pública. 2005; 31(4).

16. Gustavo Benítez. Calidad de datos: factor crítico[sitio en Internet. [citado 25 Ene 2007]. Disponible en http://www.pyme.com.mx/http://www.pyme.com.mx/

17. Gran M, Castañeda I. Estadísticas Sanitarias. Temas Docentes [sitio en Internet]. [citado 25 Ene 2007]. Disponible en :

http://www.sld.cu/galerias/doc/sitios/dne/folleto bioestadistica.doc

18. Morcillo Rubio MP. Alarmas del Sistema [sitio en Internet]. [citado 25 Ene 2007]. Disponible en:

http://www. sameens. dia.uned.es/Trabajos3/T7A/MorcilloRubioMP/Alarmas. htm

19. CDC. Update: Changes in Notifiable Disease Surveillance Data-United States, 1992-1993 October 29, 1993. MMWR [sitio en Internet]. [citado 2 Oct 2007]; 42(42). Disponible en :

http://www.cdc.gov/mmwr/preview/mmwrhtml/00022112.htm

20. Oliva M. [Encefalitisporarbovirus] Virus del Nilo: vigilancia e intervención Ministerio de Salud [sitio en Internet]. [citado 25 Ene 2007]. Disponible en: http://listas.secyt.gov.ar/pipermail /encefalitisporarbovirus/2006-April/000006.html

21. Troyes L, Fuentes L, Troyes M, Canelo D. Etiología del Síndrome febril agudo en la provincia de Jaén, Perú 2004-2005. Rev Peru Med Exp Salud Publica [serie en Internet]. 2006[citado 25 Ene 2007];23(1). Disponible en: http://sisbib.unmsm.edu.pe/Bvrevistas/Medicina_Experimental L23 n1/pdf/a02v23n1.pdf

22. Maravall A, Luna C. Un nuevo método para el control de calidad de los datos en series temporales. Boletín económico-Banco de España [sitio en Internet]. 1999[ citado 2 Oct 2007]; (5). Disponible en: http://dialnet.unirioja.es/servlet/articulo?codigo $=240148$

23. Quirós J. Control de Calidad de los datos en Series de Tiempo Económicas[sitio en Internet]. [citado 2 Oct 2007]. Disponible en: 
http://www.bccr.fi.cr/ndie/Documentos/DIE-01-2002-NT -

CONTROL\% 20DE\% 20CALIDAD\% 20DE\% 20LOS\% 20DATOS\% 20EN\% 20SERIES\% 20

DE\% 20TIEMPO.pdf

24. Aguilera JF, Paget WJ, Van der Velden J. Desarrollo de un protocolo para evaluar la calidad de los datos de la gripe recogidos por los médicos centinela en Europa. Euro Surveill [serie en Internet]. 2002[citado 6 Oct 2007]; 7(11).

Disponible en: http://www.eurosurveillance.org/em/v07n11/0711-222.asp

25. Díaz Tito J. Evaluación de la Calidad del Programa de Control de la Tuberculosis en el Municipio La Lisa. Ciudad Habana. Año 2001. Segunda Parte. Boletín Epidemiológico Semanal Del IPK [sitio en Internet]. 2002[citado 2 Oct 2007]; 12(37). Disponible en URL: http://www.ipk.sld.cu/bolepid/bol37-02.htm

26. Guanche Garcell. H, García Arbola E, Gutiérrez García F. Morbilidad y letalidad hospitalaria por asma bronquial. Rev Cubana Med [serie en Internet]. 2005[citado 2 Oct 2007]; 44(3-4). Disponible en: http://bvs.sld.cu/revistas/med/vol44_34_05/med033-405.htm

27. Almenares K. Técnicas de control de la calidad industrial aplicadas a la vigilancia en salud [tesis]. La Habana: Escuela Nacional de Salud Pública; 2005.

28. Torrescano A, García JJ. Canal Endémico: Concepto[sitio en Internet]. [citado 25 Ene 2007]. Disponible en :

http://uiip.facmed.unam.mx/deptos/salud/spdos/presentaciones/canal_endemico/

29. Jiménez Acosta S. Errores en que se puede incurrir en los Sistemas de Vigilancia Alimentaria y Nutricional. Rev Cubana Aliment Nutr [serie en Internet]. 2001[ citado 3 Oct 2007]; 15(1). Disponible en :

http://www.bvs.sld.cu/revistas/ali/vol15_1_01/ali10101.htm

Recibido: 18 de Oct de 2007.

Aprobado: 25 de mayo de 2009.

Gisele Coutin Marie. Unidad Nacional de Análisis y Tendencias en Salud Nacional/ Ministerio de Salud Pública. La Habana, Cuba.

E-mai: gisele.coutin@infomed.sld.cu 